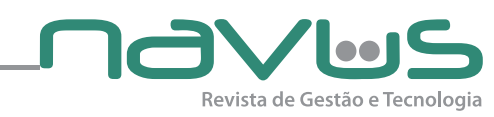

\title{
Business intelligence na auditoria geral do Estado do Rio de Janeiro: aplicabilidade e conhecimento
}

Num mundo globalizado com constante avanço tecnológico, cada vez mais as instituições necessitam de recursos relevantes para tomada de decisões. A situação ocorre em diferentes áreas de atuação e níveis hierárquicos, sendo estes recursos necessários para gestores e técnicos. Para atendimento aos usuários, frequentemente são realizadas demandas ao setor de Tecnologia da Informação (TI), que consomem tempo e impactam no custo de oportunidade dos trabalhadores dessa área. Uma forma de reduzir a demanda junto aos profissionais da TI é pela utilização do Business Intelligence (BI), que permite modelagem de dados com ferramentas analíticas e sintéticas de acordo com o desejo das instituições. Para o êxito na utilização do BI, é indispensável que os usuários detenham conhecimento das utilidades e ferramentas disponíveis. Realizamos nossa pesquisa no âmbito da Auditoria Geral do Estado do Rio de Janeiro (AGE/RJ). De acordo com Decreto Estadual 43.463/2012, AGE/RJ é o órgão central do sistema de controle interno do Poder Executivo do Estado do Rio de Janeiro e atua nas atividades de fiscalização contábil, financeira, orçamentária, operacional e patrimonial da Administração Direta e Indireta, quanto à observância da legalidade, legitimidade, economicidade na aplicação dos gastos públicos e constitui etapa final do controle interno. Em sua estrutura hierárquica, a AGE/RJ é composta pelo Auditor Geral, seguido de Superintendentes e Coordenadores, segundo e terceiro escalões, respectivamente. Diante das atribuições legais do órgão, estudamos acerca do nível de conhecimento sobre BI dos segundo e terceiro níveis hierárquicos da AGE/RJ. O objetivo da pesquisa é identificar o nível de conhecimento de BI dos Superintendentes e Coordenadores de AGE/RJ. Foi aplicado questionário com perguntas fechadas e escala likert. 0 resultado apresentado mostra uma relação entre as funções exercidas dos servidores e o conhecimento sobre BI. A pesquisa serve como parâmetro para necessidade de capacitação de grupo de servidores com características semelhantes.

Palavras-Chave: Business Intelligence. Aplicabilidade. Gestão do conhecimento.

\footnotetext{
${ }^{1}$ Mestrando em Gestão e Estratégia. Universidade Federal Rural do Rio de Janeiro (UFRRJ), leonsysad@yahoo.com.br;

${ }^{2}$ Doutorando em Ciência Política, Instituto Universitário de Pesquisa do Rio de Janeiro (IUPERJ), roliveira@fazenda.rj.gov.br;

${ }^{3}$ Mestrando em Administração e Desenvolvimento Empresarial, Universidade Estácio de Sá (UNESA), thiagoclcontabil@yahoo.com.br;

${ }^{4}$ Mestrando em Ciências Contábeis, Universidade Estadual do Rio de Janeiro (UERJ), vmsmiranda@yahoo.com.br.
} 


\section{INTRODUÇÃO}

Os avanços tecnológicos têm impulsionado a utilização de Tecnologia da Informação (TI) pelas organizações, que por sua vez buscam otimizar a realização de atividades. Na administração pública não é diferente, pois, tem sido demandado dos setores de TI a projeção de softwares que auxiliem na modelagem de dados com ferramentas analíticas e sintéticas de acordo com o desejo das instituições. Todavia, para o êxito na utilização do BI, é imprescindível que os usuários detenham conhecimento das utilidades e ferramentas disponíveis.

Nesse sentido, buscou-se identificar o nível de conhecimento de BI dos servidores da Auditoria Geral do Estado do Rio de Janeiro. Os dados foram coletados nos meses de julho e agosto de 2013, utilizando-se a metodologia survey.

A AGE/RJ é o órgão central do sistema de controle interno do Poder Executivo do Estado do Rio de Janeiro e pertence à estrutura da Secretaria de Estado de Fazenda, que possui atribuições de fiscalização contábil, financeira, orçamentária, operacional e patrimonial da Administração Direta e Indireta, quanto à observância da legalidade, legitimidade, economicidade na aplicação dos gastos públicos e constitui etapa final do controle interno.

Espera-se que os resultados obtidos possam contribuir para construção de parâmetro para capacitação de grupo de servidores com características semelhantes, assim como para investigação da percepção de profissionais formados em áreas do conhecimento fora da TI, pois, na AGE, a formação dos servidores não é multidisciplinar. Assim, é possível que os auditores utilizem a ferramenta sem reconhecê-la como Business Intelligence (BI).

\section{REFERENCIAL TEÓRICO}

O referencial teórico deste estudo foi dividido em duas subseções, a primeira para apresentar o conceito e a técnica da consolidação de demonstrações contábeis; a segunda, para apresentar os conceitos de BI.

De acordo com Heidemann e Salm (2009), a eficiência e a qualidade dos serviços públicos dependem muito da relação direta e transparente entre os prestadores de serviço e os respectivos beneficiários. A busca por melhorias é uma constante para empresas e a administração pública, pois, dessa forma, permitese mensurar os resultados alcançados.

Segundo Serra e Carneiro (2011), colocar a casa em ordem é o primeiro passo rumo à futura responsabilização dos gestores e cobrança acerca da eficiência da máquina, pois de nada adiantaria fortalecer o controle externo, ou incluir a vigilância popular, se não ocorrer uma organização estruturada para dar respostas à sociedade e ao controle. Diante do arcabouço legal e da quantidade de dados e informações passadas na gestão pública, o uso da tecnologia tornou-se vital para o desempenho satisfatório da administração pública.

Para entender as necessidades informacionais dos usuários, atualmente não se pode desconsiderar a tecnologia da informação e seus respectivos recursos na utilização dos sistemas de informações, e na consequente geração e transmissão do recurso informação (REZENDE; ABREU, 2003). Segundo percepção de 0' Brien (2004), a referida tecnologia pode auxiliar a organização a melhorar a eficiência de seus processos, tomadas de decisões, e, com isso, fortalecer uma posição competitiva em seu mercado.

Dada a sua ascensão e relevância para as organizações, é importante ressaltar, conforme Keen (1993), que o conceito de TI é mais abrangente do que os sistemas de informação ou conjunto de hardware e software, ou seja, a TI, envolve, além dos referidos sistemas, também os aspectos humanos, administrativos e organizacionais. Nessa linha, McGee e Prusak (1994), defendem que o recurso informação deve ser considerado o maior potencial de retorno às organizações (públicas e privadas) e não somente a tecnologia, como muitos autores defendem.

A tecnologia da informação, considerando-se especialmente as ferramentas de BI, serve de apoio ao sistema existente na empresa, a partir de meios que permitam que os dados sejam armazenados de forma segura e transformados em informações úteis que devem ser comunicadas a qualquer tempo, de forma apropriada, a seus usuários. Para isso, a empresa tem o apoio do sistema de controle interno, que existe justamente para proporcionar a segurança dos registros, das informações e do próprio sistema informatizado.

Partindo do raciocínio de que a TI existe para flexibilizar e tornar dinâmicos os sistemas existentes e auxiliar na veiculação da informação entre os usuários que dela necessitam para realizar as suas atividades, e que esses sistemas compõem a fonte de dados da empresa. Analisam-se, a seguir, as ferramentas utilizadas 
na geração, no tratamento, e na comunicação da informação: BI.

As ferramentas de BI podem fornecer uma visão sistêmica do negócio e ajudar na distribuição uniforme dos dados entre os usuários, sendo seu objetivo principal transformar grandes quantidades de dados em informações de qualidade para tomada de decisões. Por meio delas, é possível cruzar dados, visualizar informações em várias dimensões e analisar os principais indicadores de desempenho empresarial (BATISTA, 2004). Essa facilidade, considerando-se as características dessas ferramentas, pode contribuir diretamente para as funções na área de controladoria na obtenção, análise e comunicação do recurso informação aos gestores, além de permitir a essa área o estreito monitoramento das atividades da empresa na sua totalidade.

Define-se BI como "guarda-chuva" conceitual, visto que se dedica à captura de dados, informações e conhecimentos que permitem às empresas competirem com maior eficiência em uma abordagem evolutiva de modelagem de dados, capazes de promover a estruturação das informações em depósitos retrospectivos e históricos, permitindo sua modelagem por ferramentas analíticas. Seu conceito é bastante abrangente e envolve todos os recursos necessários para o processamento e disponibilização da informação para o usuário (BARBIERI, 2001).

Esse guarda-chuva BI, figura desde simples planilhas eletrônicas, geradoras de consultas e relatórios, até soluções mais aprimoradas que incluem ferramentas mais sofisticadas (ex.: Data Mining). Qual ferramenta utilizar vai depender basicamente do poder de investimento da organização, pois de uma maneira ou de outra, todas atendem ao básico, ou seja, todas agilizam a obtenção de dados; porém, conforme o aprimoramento da ferramenta, o valor a ser investido naturalmente aumenta. Importante ter em mente, para quem desenvolve em ambiente de BI, é que seja adotada ferramenta que permita obter detalhes das bases volumosas de dados, com o custo de propriedade menor tanto quanto possível e mantendo um desempenho maior, tanto quanto permitido.

A necessidade de se implantar um ambiente de BI é fundamental não somente às grandes corporações, mas também, às pequenas e médias, pois é necessário cada vez mais ampliar o conhecimento do negócio, de modo a estar atento às variações do mercado, da concorrência e das oportunidades na área de atuação, qualquer que seja ela, governo, indústria, educação.

A administração pública, em particular a auditoria governamental, busca, mediante as ferramentas de BI, apontar indicadores de performance e tendência com diferentes graus de sintetização, capazes de auxiliála na condução dos trabalhos de auditoria. Nessa linha, MCGeever (2000) apresenta uma outra visão do conceito de BI, referente ao tipo de informação segmentada que o gestor de um ramo de negócios procura. Fazendo o uso do BI, o auditor pode formatar as suas próprias informações, assim como pode conectá-las a outras a fim de obter uma melhor análise e um melhor resultado com o seu uso, isto é, ele pode tornarse mais independente na busca das informações mais adequadas com minimização do uso de relatórios distintos.

Segundo Bolieiro (2008), no mund o de tempestade de informações, torna-se necessário conseguir filtrar as informações, e o BI pode ajudar em dois aspectos fundamentais: reduzir custos (financeiro e temporal) e aumentar receitas. Isso pode ser realizado mediante otimização de processos, por meio da análise de dados, melhora ou criação de novos produtos.

BI não significa somente ceder informações às pessoas, mas gerar apoio a qualquer decisão corporativa, de todos os departamentos e níveis hierárquicos. 0 próprio uso de um sistema de BI tem o potencial de prover novas formas de entender os dados que a empresa dispõe; portanto, novas informações, capazes de gerar um novo entendimento, ou conhecimento, para o usuário final. 0 BI tem uma forte ligação com a vertente tecnológica da gestão do conhecimento (PARREIRA; MATHEUS, 2004).

As ferramentas de BI auxiliam os servidores que atuam em Auditorias Governamentais com oferecimento de suporte adequado e mapeamento de riscos à administração pública de diferentes formas, como segmentação do mercado, análises estatísticas, inventários, entre outras infinidades de aplicações. Essas informações são disponibilizadas por meio de relatórios à equipe técnica, a fim de que determinem as tendências, satisfaçam necessidades e dimensionem melhor a tomada de decisão.

O Quadro 1 apresenta as principais vantagens e desvantagens da aplicação do BI na administração pública. 


\begin{tabular}{|l|l|l|}
\hline \multicolumn{2}{|c|}{ Quadro 1 - Aplicabilidade do Business Intelligence (BI) } \\
\hline Aplicação do BI & \multicolumn{1}{|c|}{ Vantagens } & \multicolumn{1}{c|}{ Desvantagens } \\
\hline & Agilidade. & $\begin{array}{l}\text { Custo. } \\
\text { Curva de aprendizado lenta por parte dos usuários. } \\
\text { Despesas adicionais com infraestrutura. } \\
\text { Insucesso após implantação gerada por falta de foco. } \\
\text { Resistência à utilização por parte dos usuários. } \\
\text { Suporte. }\end{array}$ \\
\hline Administração Pública & $\begin{array}{l}\text { Confiabilidade e segurança. } \\
\text { Gerenciamento de recursos operacionais. } \\
\text { Melhor planejamento. } \\
\end{array}$ & Mapeamento de risco. \\
& Tomada de decisão eficaz. \\
Transparência. & \\
\hline
\end{tabular}

Fonte: Elaboração própria (2013)

\section{METODOLOGIA}

Os dados analisados neste estudo foram coletados em julho e agosto de 2013, utilizando-se a metodologia survey, que, segundo Hair Júnior et al (2005, p. 157), "é um procedimento para coleta de dados primários a partir de indivíduos", cujos dados podem variar entre crenças, opiniões, experiências do indivíduo e outros.

Ainda conceituando, Cooper e Schindler (2008) explicam que survey é um processo de mensuração apropriado para o levantamento de informações em uma entrevista altamente estruturada, utilizando geralmente um questionário, com ou sem o envolvimento do entrevistador. Nesse contexto, geralmente, utiliza-se a metodologia quantitativa de modo a se investigar se uma determinada população possibilita a inferência de conclusões sobre ela, por meio de observações de uma pequena amostra dessa população (COLLIS; HUSSEY, 1997).

Aqui a população é composta por Analistas de Controle Interno e Extra-Quadro, que trabalham na Auditoria Geral do Estado do Rio de Janeiro (AGE/RJ), órgão integrante do Poder Executivo do Governo do Estado, vinculado à Secretaria de Estado de Fazenda, para os quais foram enviados os questionários da pesquisa, contemplando questões fechadas e abertas de modo a se conseguir revelar informação sobre o perfil dos auditores e o conhecimento deles sobre o objeto investigado por esta pesquisa, com o auxílio do "Google Drive", tratando-se, portanto, de survey eletrônica.

0 critério foi adotado devido à sua clareza e pela oportunidade e facilidade quanto à obtenção de dados no órgão participante da survey, sobretudo pelo fato de os pesquisadores trabalharem na AGE/ RJ. Basicamente, o que se pretende investigar e a percepção dos auditores, em termos de mensuração de frequências, quanto ao seu conhecimento e utilização de BI, que, aqui, é uma ferramenta de busca e mineração de dados de modo a possibilitar e facilitar a coleta de evidências para basear a opinião do auditor que será expressa pelos relatórios e pareceres a respeito das demonstrações contábeis e outros contextos.

Na AGE/RJ, a formação dos servidores não é multidisciplinar, todos possuem formação na área da Ciência Contábil. Assim, é possível que os auditores utilizem a ferramenta sem reconhecê-la como BI.

Do exposto, a amostra aqui analisada é do tipo não probabilística por conveniência e proximidade, visto que os participantes foram escolhidos pelo fato de estarem disponíveis. Dos 120 questionários enviados, 77 foram respondidos, obtendo-se, desse modo, uma fração significativa para a análise, 64\% da população.

Por fim, na seção seguinte, serão apresentados os resultados obtidos na survey.

\section{RESULTADOS}

Dos resultados obtidos, verificou-se que a amostra é composta por maioria de homens (53\%) e 48,05\% encontram-se na faixa etária compreendida entre 25 e 40 anos, conforme ilustrado na Tabela 2. Dentre os respondentes, $45 \%$ possuem mais de quinze anos de experiência. Ao se considerar o tempo de exercício profissional em Auditoria, a maioria (35\%) atua no ramo no período de um a cinco anos. 


\begin{tabular}{|c|c|c|c|c|c|c|}
\hline \multirow{2}{*}{ Faixa Etária } & \multicolumn{2}{|c|}{ Feminino } & \multicolumn{2}{|c|}{ Masculino } & \multicolumn{2}{|c|}{ Total } \\
\hline & $\mathrm{n}=$ & $\%$ & $n=$ & $\%$ & $n=$ & $\%$ \\
\hline Total & 36 & 100,00 & 41 & 100,00 & 77 & 100,00 \\
\hline Abaixo de 25 anos & 2 & 5,55 & 2 & 4,87 & 4 & 5,19 \\
\hline De 25 a 40 anos & 18 & 51,00 & 19 & 46,34 & 37 & 48,05 \\
\hline De 41 a 55 anos & 14 & 38,88 & 17 & 41,46 & 31 & 40,25 \\
\hline Acima de 55 anos & 2 & 5,55 & 3 & 7,31 & 5 & 6,49 \\
\hline
\end{tabular}

Fonte: Elaboração própria (2013)

O quadro funcional da Auditoria Geral do Estado é composto por servidores de carreira, aprovados em concurso público, e servidores denominados extraquadros, os quais são ocupantes de cargo em comissão. Os cargos em comissão são aqueles destinados ao livre provimento e exoneração, de caráter provisório, destinando-se apenas às atribuições de direção, chefia e assessoramento, podendo recair ou não em servidor de carreira do Estado.

A amostra da pesquisa foi composta por 66 servidores de carreira e onze servidores extraquadros. Identificou-se, também, que, do total de respondentes, 49 possuem cargos comissionados, sendo os de maiores níveis hierárquicos (Superintendente, Coordenador e Supervisor) ocupados por servidores de carreira, e as funções de assessoramento (Assessor, Assistente e Secretária) são exercidas por servidores extraquadro, conforme se depreende da Tabela 2.

\begin{tabular}{|c|c|c|c|}
\hline Cargo em Comissão & Extraquadros & Servidor de Carreira & Total Geral \\
\hline Total & 11 & 66 & 77 \\
\hline Superintendente & - & 3 & 3 \\
\hline Coordenador(a) & - & 14 & 14 \\
\hline Assessor(a) Setorial & 1 & 3 & 4 \\
\hline Supervisor(a) & - & 16 & 16 \\
\hline Auditor Interno ou equivalente & 1 & 3 & 4 \\
\hline Assistente & 5 & 1 & 6 \\
\hline Secretária & 2 & - & 2 \\
\hline Não ocupante de Cargo em Comissão & 2 & 26 & 28 \\
\hline
\end{tabular}

Fonte: Elaboração própria (2013)

Quanto à escolaridade, 94\% dos respondentes possuem nível superior. Quanto à titularidade, considerando aqueles que ainda estejam cursando, pelo menos $60 \%$ possuem pós-graduação, $9 \%$ mestrado e $2 \%$ doutorado. Verificou-se que os servidores que ingressaram por intermédio de concurso público têm graduação em Ciências Contábeis em razão da natureza das atividades a serem desempenhadas; todavia, foi possível perceber que 5\% têm alguma formação relacionada com TI e $27 \%$ já participaram de alguma capacitação relacionada à temática de TI.

Ao investigar a percepção dos servidores no que concerne a conhecimento e utilização de BI, percebeu-se que, apesar de $80 \%$ informarem não conhecer ferramenta de BI que possa apoiar na execução de atividades, todavia, 89\% utilizam o SIG, que é uma ferramenta de BI. Ademais, a maioria (49\%) dos respondentes afirmou utilizá-lo semanalmente, $13 \%$ quinzenalmente, $18 \%$ mensalmente, $8 \%$ semestralmente, $4 \%$ anualmente e apenas $8 \%$ nunca o utilizaram.

Ainda relativamente à utilização do SIG, 34\% informaram que a maior frequência de utilização do sistema para execução de suas atividades refere-se à elaboração de Relatórios de Auditoria, 30\% informaram que o utilizam mais para execução dos trabalhos de auditoria, 18\% para o planejamento das atividades e $9 \%$ para o monitoramento. 


\begin{tabular}{|c|c|c|c|c|c|c|}
\hline \multirow{2}{*}{$\begin{array}{l}\text { Frequência de uso } \\
\text { do SIG }\end{array}$} & \multicolumn{6}{|c|}{ Etapas } \\
\hline & Planejamento & Execução & $\begin{array}{c}\text { Elaboração de } \\
\text { Relatórios }\end{array}$ & Monitoramento & Não utiliza o SIG & Total Geral \\
\hline Total & 14 & 23 & 26 & 7 & 7 & 77 \\
\hline Anualmente & - & 1 & - & 1 & - & 3 \\
\hline Mensalmente & 2 & 4 & 6 & 2 & - & 14 \\
\hline Quinzenalmente & 1 & 3 & 5 & 1 & - & 10 \\
\hline Semanalmente & 9 & 13 & 14 & 2 & - & 38 \\
\hline Semestralmente & 2 & 2 & 1 & 1 & - & 6 \\
\hline Nunca & - & - & - & - & 7 & 6 \\
\hline
\end{tabular}

Fonte: Elaboração própria (2013)

Quando questionados sobre o maior problema encontrado na execução das atividades de auditoria, 34\% informaram o grande volume de dados para analisar, 31\% alegaram a falta de informações concretas e confiáveis, $14 \%$ apontaram o tempo para levantar dados, $10 \%$ apontaram a falta de dados confiáveis e apenas $5 \%$ consideraram a insegurança na formação da opinião de auditoria como maior problema.

Quanto à natureza das análises realizadas utilizando TICs, apenas 6\% alegaram não utilizá-la, 69\% fazem análises patrimoniais e orçamentárias empregando TI e 65\% avaliam aspectos financeiros das Demonstrações Contábeis e Prestações de Contas por intermédio de TICs. Esses resultados apontam a importância de TI para o exercício de auditoria.

Houve também um questionamento acerca da importância percebida pelos servidores a propósito da utilização de ferramentas de TI para a execução das atividades de auditoria, considerando escala numérica de 0 a 10, derivada de Likert (onde zero significa nenhuma importância e dez total importância), e apurouse que a nota com maior frequência equivale a 10, já a média de valores das respostas corresponde a 9, conforme ilustrado no Gráfico 1.

Buscou-se conhecer a opinião dos respondentes acerca da importância da ferramenta de BI para apoio à execução de atividades e verificou-se que apenas 7\% não acreditam ser importante, 47\% afirmam ser importante e $46 \%$ não têm opinião formada.

\section{CONCLUSÃO}

O objetivo da pesquisa era conhecer o nível de conhecimento de BI entre os servidores da Auditoria Geral do Estado e verificou-se que, apesar de $46 \%$ da amostra não ter opinião formada sobre a importância de BI e 80\% informarem não conhecer ferramenta de BI que auxilie na execução de atividades, 89\% utilizam o SIG, que é uma ferramenta de BI.

A nota média atribuída pelos servidores da AGE/RJ no que se refere à importância de TI para execução de atividades de auditoria foi equivalente a 9 e apenas 7\% não consideram que ferramentas de BI apoiem a realização de suas atividades.

Portanto, as ferramentas de TI são percebidas com grande valor pelos profissionais da Auditoria Geral do Estado, dentre os quais o uso de ferramenta de BI é bastante frequente, pois $80 \%$ acessam o SIG pelo menos mensalmente, embora apenas $20 \%$ informaram conhecer ferramentas de BI.

Tais resultados comprovam a hipótese de que profissionais sem formação na área de TI, embora utilizem ferramentas de BI e considerem-nas importantes para execução de atividades, nem sempre conseguem associar os termos técnicos especializados ao domínio de conhecimento em relação a um contexto.

Por fim, a presente pesquisa está limitada à investigação de apenas 77 servidores que atuam na atividade de auditoria e trabalham em um órgão público, razão pela qual os resultados aqui revelados não podem ser generalizados. Assim, pesquisas futuras poderão verificar a percepção da importância de ferramentas de TI em outras organizações com carreiras multidisciplinares, de modo a investigar se o fenômeno lá é mitigado. 


\title{
BUSINESS INTELLIGENCE IN AUDIT GENERAL OF THE STATE OF RIO DE JANEIRO: APLICABILITY AND KNOWLEDGE
}

\begin{abstract}
In a globalised world with constant technological advancement increasingly institutions require resources relevant to decision-making. The situation occurs in different practice areas and hierarchical levels, being necessary for managers and technicians. For service users, are often undertaken demands to the sector of information technology (it), which consume time and impact on the opportunity cost of workers of that area. One way to reduce the demand on IT workers is through the use of Business Intelligence (BI), which allows data modeling with analytical and synthetic tools according to the desire of the institutions. For the successful use of BI is necessary that users hold knowledge of utilities and tools available. We conduct our research in the scope of the Auditor General of the State of Rio de Janeiro (AGE/RJ). According to State Decree 43.463/2012, AGE/RJ is the central organ of the internal control system of the Executive power of the State of Rio de Janeiro and serves on the supervisory activities accounting, financial, budgetary, operational and asset of the direct and indirect administration, as to the observance of legality, legitimacy, economy in the implementation of public expenditure and constitutes the final stage of internal control. In their hierarchical structure the AGE/RJ is composed by the Auditor General, followed by Superintendents and engineers, second and third places, respectively. Under the legal duties of the organ studied about the level of knowledge of BI of the second and third hierarchical levels of AGE/RJ. The goal of the research is to identify the level of knowledge of BI of the civil servants of the AGE/RJ. A questionnaire was applied with likert scale and close-ended questions. The result presented shows a relationship between the duties of servers and the knowledge about BI. The research serves as parameter for the need of a training group of civil servants with similar characteristics.
\end{abstract}

Keywords: Business Intelligence. Applicability. knowledge management. AGE/RJ

\section{REFERÊNCIAS}

BARBIERI, Carlos. BI - Business Intelligence: Modelagem e Tecnologia. Rio de Janeiro: Axcel Books, 2001.

BATISTA Emerson de Oliveira. Sistemas de informação. São Paulo: Saraiva, 2004.

BOLIEIRO, Flávio. Business Intelligence - Saiba como as ferramentas de BI permitem reunir, armazenar e analisar os dados de companhias de todos os portes. Revista PC WORLD Extra, São Paulo, Redação da PC WORLD, p. 4-7, 2008. Disponível em:<http://lt.idg.com.br/oracle/pcw_extra_oracle.pdf >. Acesso em: 25 jul. 2013.

COLLIS, Jili; HUSSEY, Roger. Business Research: a practical guide for undergraduate and postgraduate students. Nova York: Palgrave Macmillan, 1997.

COOPER, Donald; SCHINDLER, Pamela. Business Research Methods. Nova York: MacGraw-Hill International Edition, 2008.

HAIR JUNIOR, Joseph et al. Fundamentos de Métodos de Pesquisa em Administração. Porto Alegre: Bookman, 2005.

HEIDEMANN, Francisco; SALM, José Francisco. Políticas Públicas e Desenvolvimento. Brasília: Editora UNB, 2009. 
KEEN, Peter G. W. Information technology and the management theory: the fusion map. IBM System Journal, Nova York, v. 32, p.17-38, 1993.

McGEE, James; PRUSAK, Laurence. Gerenciamento estratégico da informação. Rio de Janeiro: Campus, 1994.

McGEEVER, C. Business Intelligence. Computerworld, Arizona, p. 54, july 2000.

O’ BRIEN, James. Sistemas de informação e as decisões gerenciais na era da Internet. São Paulo: Saraiva, 2004.

PARREIRA, Fernando. S.; MATHEUS, Renato. F. Inteligência Empresarial versus Business Intelligence: Abordagens complementares para o apoio à tomada de decisão no Brasil. Programa de Pós-Graduação em Ciência da Informação (PPCGI), Universidade Federal de Minas Gerais (UFMG), KMBrasil2004 - Congresso Anual da Sociedade Brasileira de Gestão do Conhecimento, 3., 2004. Anais... Disponível em: <http://www. fernando.parreiras.nom.br/publicacoes/ie_bi.pdf> Acesso em: 25 jul. 2013.

REZENDE, Denis A.; ABREU, Aline F. Tecnologia da informação aplicada a sistemas de informação empresariais. São Paulo: Atlas, 2003.

SERRA, Rita de Cassia C.; CARNEIRO, Ricardo. As Interfaces Entre o Controle Social e os Controles Externo e Interno na Administração Pública Brasileira. Encontro da ANPAD, 34., 2011. Anais do XXXIV Encontro da ANPAD 2011. Rio de Janeiro: ANPAD, 2011.. Disponível em: <http://www.anpad.org.br/diversos/ trabalhos/EnANPAD/enanpad_2011/APB/2011_APB1827.pdf.> Acesso em: 25 jul. 2013. 